\title{
Visco-Elastic Effects on Convection Flow in a Vertical Rotating Channel Partially Filled with a Porous Medium
}

\author{
Rita Choudhury \\ Department of Mathematics \\ Gauhati University \\ Guwahati, Assam, India
}

\author{
Saswati Purkayastha \\ Department of Mathematics \\ Gauhati University \\ Guwahati, Assam, India
}

\begin{abstract}
A theoretical investigation of the influence of radiation on natural convection flow of an electrically conducting viscoelastic fluid in a vertical channel partially filled by a porous medium with high porosity has been presented. It is assumed that the conducting fluid is gray, emitting-absorbing radiation, and non-scattering medium. The visco-elastic fluid is characterized by Walters liquid (Model B'). The infinite vertical porous plates of the channel are subjected to constant injection and suction velocity respectively. The entire system rotates about the axis normal to the plates with a uniform angular velocity. The perturbation scheme has been used to solve the governing equations of the fluid motion. The approximate solutions for velocity and temperature fields have been derived and the effects of the Prandtl number, Grashof number, radiation-conduction parameter (Stark number), rotation parameter, magnetic field and permeability of the porous medium on the velocity field, temperature field and Nusselt number have been discussed and illustrated graphically in possible cases. The practical use of this problem can be seen in heating of buildings, cooling electronic components and drying several types of agriculture products grain and food.
\end{abstract}

\section{General Terms}

Visco-elastic.

\section{Keywords}

Visco-elastic, permeability, porous medium, partially filled, Walters liquid (Model B').

\section{INTRODUCTION}

The study of flow through a porous medium could be very practicable in many applications in movement of water and oil inside the earth, flow of river through porous banks, chemical engineering for filtration and purification process, petroleum technology to study the movement of natural gas and in the fields of agriculture engineering to study the underground water resources.

Convective flow in channels driven by temperature differences of bounding walls have been analyzed and reported by many researchers because of its immense applications in many engineering branches. In vertical slots, free convection flows have been discussed by Aung et al. [1], Burch et al. [2], Buhler [3], Weidman and Medina [4]. Sanyal and Adhikari [5] have studied effects of radiation on MHD vertical channel flow. Chauhan and Jain [6] have measured three dimensional MHD steady flow of a viscous incompressible fluid over a highly porous layer. Normal mode analysis of the fully developed free convection flow in a vertical slot with open to capped ends has investigated by
Magyari [7]. The effect of uniform suction/ blowing on heat transfer of magnetohydrodynamic Hiemenz flow through porous media has explained by Yih [8]. Al-Nimr and Khadrawi [9] have analyzed transient free convection fluid flow in domains partially filled with porous media. Alkam et al. [10] have studied on forced convection in channels partially filled with porous substrates. Convection regime flow in a vertical slot: Continuum of solutions from capped to open ends has been investigated by Weidman [11]. Kim et al. [12] has considered effect of wall conduction on free convection between asymmetrically heated vertical plates, uniform wall heat flux. Effect of an electromagnetic field on natural convection in an inclined porous layer have examined by Bian et al. [13]. Chang and Chang [14] have reflected on mixed convection in a vertical parallel plate channel partially filled with porous media of high permeability. Fully developed free convection in open-ended vertical channels partially filled with porous material has been discussed by AlNimr and Haddad [15]. Chauhan et al. have analyzed [16] Radiation effects on natural convection MHD flow in rotating vertical porous channel partially filled with a porous medium.

Non-Newtonian fluid flow plays important roles in several industrial manufacturing processes. An example of such nonNewtonian fluids includes drilling mud, polymer solutions or melts, certain oils and greases and many other emulsions. Some of the typical applications of non-Newtonian fluid flow are noticed in the drilling of oil and gas wells, polymer sheet, extrusion from a dye, glass fiber and paper production, drawing of plastic films, waste fluids etc. The applications of the mechanisms of non-Newtonian fluid flows in modern technology and industries have attracted the researchers in a large scale. Authors like Abel et al. [17], Sonth et al. [18], Abel et al. [19], Choudhury et al. [20], [21], [22], [23] and [24] have analyzed some problems of physical interest in this field.

The purpose of the present study is to analyze the effects of natural convection flow in a vertical rotating channel partially filled with porous medium.

The constitutive equation for Walters liquid (Model B') is

$$
\sigma_{i k}=-p g_{i k}+\sigma_{i k}^{\prime}, \sigma^{\prime i k}=2 \eta_{0} e^{i k}-2 k_{0} e^{\prime i k}
$$

where $\sigma^{\mathrm{ik}}$ is the stress tensor, $\mathrm{p}$ is isotropic pressure, $g_{i k}$ is the metric tensor of a fixed co-ordinate system $\mathrm{x}^{\mathrm{i}}, \mathrm{v}_{\mathrm{i}}$ is the velocity vector, the contravarient form of $\mathrm{e}^{\prime \mathrm{ik}}$ is given by

$e^{\prime i k}=\frac{\partial e^{i k}}{\partial t}+v^{m} e^{i k}, v^{k}{ }_{, m} e^{i m}-v^{i}{ }_{, m} e^{m k}$ 
It is the convected derivative of the deformation rate tensor $\mathrm{e}^{\mathrm{ik}}$ defined by

$$
2 e^{i k}=v_{i, k}+v_{k, i}
$$

Here $\eta_{0}$ is the limiting viscosity at the small rate of shear which is given by

$\eta_{0}=\int_{0}^{\infty} N(\tau) d \tau$ and $k_{0}=\int_{0}^{\infty} \tau N(\tau) d \tau$

$\mathrm{N}(\tau)$ being the relaxation spectrum as introduced by Walters [25], [26]. This idealized model is a valid approximation of Walters liquid (Model B') taking very short memories into account so that terms involving

$$
\int_{0}^{\infty} t^{n} N(\tau) d \tau, \quad n \geq 2
$$

have been neglected.

\section{PROBLEM FORMULATION}

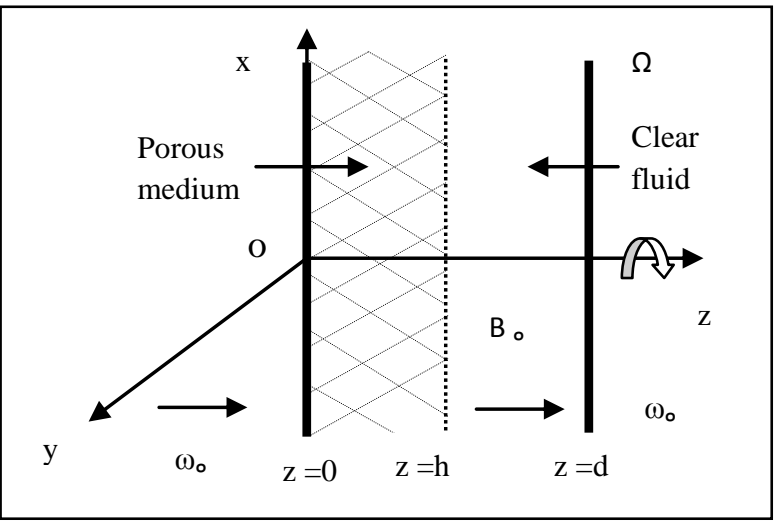

Figure 1: Physical model of the problem

We consider the unsteady MHD free convective flow in a vertical parallel porous plate channel partially filled with a porous material and partially with a clear electrically conducting fluid. The entire system rotates about the axis normal to the plates with uniform angular velocity $\Omega$. The channel is of width $\mathrm{d}$ and the thickness of the porous medium is $\mathrm{h}$. A Cartesian coordinate system is assumed and $\mathrm{z}$-axis is taken normal to the plates while $\mathrm{x}$ and $\mathrm{y}$ axes, respectively are in the upward and perpendicular directions on the plate $\mathrm{z}=0$. Two vertical plates are situated at $\mathrm{z}=0$ and $\mathrm{z}=\mathrm{d}$. The origin is taken at the plate, $z=0$ and the channel is oriented vertically upward along $\mathrm{x}$-axis. Plates are infinite in extent in $\mathrm{x}$ and $\mathrm{y}$ directions. These plates are subjected to a constant injection velocity $\left(\omega_{0}\right)$ at one plate $(\mathrm{z}=0)$ and the same constant suction velocity $\left(\omega_{0}\right)$ at the other plate $(\mathrm{z}=\mathrm{d})$. A uniform magnetic field $\mathrm{B}_{\mathrm{o}}$ is applied along an axis normal to the plates ( $\mathrm{z}$-axis) and the entire system rotates about this axis. It is assumed that the magnetic Reynolds number is very small, so that induced magnetic field is neglected (Cowling [27]).

We denote the velocity components $\bar{u}, \bar{v}, \bar{w}$ in porous medium region and $u^{\prime}, v^{\prime}, w^{\prime}$ in clear fluid region, in the $\mathrm{x}, \mathrm{y}, \mathrm{z}$ directions respectively. $\bar{T}$ denotes temperature in the porous region, $T^{\prime}$ denotes temperature in the clear fluid region, and $t$ denotes time. Since the channel plates are infinite in extent, velocity and temperature components depend only on $z^{\prime}$ and $t^{\prime}$, and further, the continuity equation gives $\bar{w}=w^{\prime}=w_{o}$ (constant).

The fluid considered here is assumed to be gray, emittingabsorbing radiation but a non-scattering medium. It is also assumed that there is radiation only from the fluid. Further it is assumed that the thermal radiation is present in the form of a unidirectional flux, transverse to the vertical plates and to describe the thermal radiative heat transfer, the Rosseland approximation is used in the energy equation. Further, the fluid and the porous structure are assumed to be in local thermal equilibrium and all the fluid properties are assumed to be constant except that the influence of the density variation with temperature is considered only in the body force term.

Using Rosseland approximation, the radiative heat transfer takes the form following Siegel and Howell [28], respectively in clear fluid and porous region as follows:

$$
q_{r}=-\frac{4 \sigma^{*}}{3 K^{*}} \frac{\partial T^{\prime 4}}{\partial z^{\prime}} ; \quad \overline{q_{r}}=-\frac{4 \sigma^{*}}{3 K^{*}} \frac{\partial \bar{T}^{4}}{\partial z^{\prime}}
$$

where, $\sigma^{*}$ is Stefan-Boltzmann constant and $K^{*}$ is mean absorption coefficient for thermal radiation.

Following Raptis [29], the temperature functions in (6) can be expressed as a linear function of temperature. Expanding $T^{\prime 4}$ and $\bar{T}^{4}$ in a Taylor series about $T_{d}$ (constant temperature of the right wall) and neglecting higher-order terms, we obtain

$$
T^{\prime 4}=4 T_{d}^{3} T^{\prime}-3 T_{d}^{4}, \quad \text { and } \quad \bar{T}^{4}=4 T_{d}^{3} \bar{T}-3 T_{d}^{4}
$$

By introducing the following non-dimensional quantities,

$$
\begin{gathered}
\eta=\frac{z^{\prime}}{d}, \alpha=\frac{h}{d}, K=\frac{K^{\prime}}{d^{2}}, \lambda=\frac{w_{0} d}{v}, t=\frac{v t^{\prime}}{d^{2}} \\
n=\frac{d^{2} n^{\prime}}{v}, U=\frac{\bar{u}}{w_{0}}, V=\frac{\bar{v}}{w_{0}}, u=\frac{u^{\prime}}{w_{0}}, v=\frac{v^{\prime}}{w_{0}}, \theta=\frac{\bar{T}-T_{d}}{T_{0}-T_{d}}
\end{gathered}
$$

in equations (6) and (7), the dimensionless governing equations in the porous medium region $\mathrm{I}(0 \leq \eta \leq \alpha)$ and the clear fluid region II $(\alpha \leq \eta \leq 1)$ for the MHD convective flow of a radiative fluid in the rotating system are respectively given as follows:

For porous region I:

$$
\begin{aligned}
& \frac{\partial U}{\partial t}+\lambda \frac{\partial U}{\partial \eta}-2 R e V=\frac{\partial^{2} U}{\partial \eta^{2}}-k\left(\frac{\partial^{3} U}{\partial t \partial \eta^{2}}+\lambda \frac{\partial^{3} U}{\partial \eta^{3}}\right) \\
&+ G r(\theta-1)-\frac{U}{K}-M^{2} U \\
& \frac{\partial V}{\partial t}+\lambda \frac{\partial V}{\partial \eta}+2 R e U=\frac{\partial^{2} V}{\partial \eta^{2}}-k\left(\frac{\partial^{3} V}{\partial t \partial \eta^{2}}+\lambda \frac{\partial^{3} V}{\partial \eta^{3}}\right)-\frac{V}{K} \\
&-M^{2} V \\
& \frac{\partial \theta}{\partial t}+\lambda \frac{\partial \theta}{\partial \eta}=\left(\frac{3 N+4}{3 N P r}\right) \frac{\partial^{2} \theta}{\partial \eta^{2}}
\end{aligned}
$$

For porous region II:

$$
\begin{aligned}
\frac{\partial u}{\partial t}+\lambda \frac{\partial u}{\partial \eta}-2 \operatorname{Rev} & =\frac{\partial^{2} u}{\partial \eta^{2}}-k\left(\frac{\partial^{3} u}{\partial t \partial \eta^{2}}+\lambda \frac{\partial^{3} u}{\partial \eta^{3}}\right) \\
& +G r(T-1)-M^{2} u
\end{aligned}
$$




$$
\begin{gathered}
\frac{\partial v}{\partial t}+\lambda \frac{\partial v}{\partial \eta}+2 R e u=\frac{\partial^{2} v}{\partial \eta^{2}}-k\left(\frac{\partial^{3} v}{\partial t \partial \eta^{2}}+\lambda \frac{\partial^{3} v}{\partial \eta^{3}}\right) \\
-M^{2} v \\
\frac{\partial T}{\partial t}+\lambda \frac{\partial T}{\partial \eta}=\left(\frac{3 N+4}{3 N P r}\right) \frac{\partial^{2} T}{\partial \eta^{2}}
\end{gathered}
$$

where,

$$
\begin{aligned}
& R e=\frac{\Omega d^{2}}{v}, \operatorname{Pr}=\frac{\mu C_{p}}{\kappa}, N=\frac{\kappa K^{*}}{4 \sigma^{*} T_{d}^{3}}, \\
& M^{2}=\frac{\sigma}{\mu} B_{0}^{2} d^{2}, G r=\frac{g \beta\left(T_{0}-T_{d}\right) d^{2}}{\nu w_{0}}
\end{aligned}
$$

Here $v, \beta, \sigma, \kappa, C_{p}, g, n^{\prime}$ denote kinematic viscosity, coefficient of volume expansion, electrical conductivity, thermal conductivity, specific heat at constant pressure, acceleration due to gravity and dimensional scalar constant respectively. $\mathrm{K}^{\prime}$ is the dimensional permeability of the porous medium, $\mathrm{K}$ is the dimensionless permeability of the porous medium, $T_{0}$ is a reference temperature and $T_{d}$ is constant temperature of the right wall.

The relevant boundary conditions in nondimensional form are given by

$$
\begin{aligned}
& \text { at } \eta=0 ; \quad U=V=0, \quad \theta=1+\varepsilon e^{n t} \\
& \text { at } \eta=\alpha ; \quad U=u, \quad V=v, \\
& \frac{\partial U}{\partial \eta}=\frac{\partial u}{\partial \eta}, \quad \frac{\partial V}{\partial \eta}=\frac{\partial v}{\partial \eta}, \quad \frac{\partial \theta}{\partial \eta}=\frac{\partial T}{\partial \eta}
\end{aligned}
$$$$
\text { at } \eta=1 ; \quad u=v=0, \quad T=
$$

Here, $\varepsilon \ll 1$ (a positive constant), and $\mathrm{n}$ is a dimensionless scalar constant.

\section{METHOD OF SOLUTION}

We solve equations (10) and (13) for temperature distribution in porous and clear fluid region. Let us assume

$$
\begin{gathered}
\theta(\eta, t)=\theta_{0}(\eta)+\varepsilon \theta_{1}(\eta) e^{n t} \\
T(\eta, t)=T_{0}(\eta)+\varepsilon T_{1}(\eta) e^{n t}
\end{gathered}
$$

Substituting (15) and (16) in equations (10) and (13) with corresponding boundary conditions for the temperature distribution, and comparing the coefficients of $e^{n t}$, we obtain

$$
\begin{aligned}
& \frac{\partial^{2} \theta_{0}}{\partial \eta^{2}}-\left(\frac{3 \lambda N P r}{3 N+4}\right) \frac{\partial \theta_{0}}{\partial \eta}=0 \\
& \frac{\partial^{2} \theta_{1}}{\partial \eta^{2}}-\left(\frac{3 \lambda N P r}{3 N+4}\right) \frac{\partial \theta_{1}}{\partial \eta}-\left(\frac{3 n N P r}{3 N+4}\right) \theta_{1}=0 \\
& \frac{\partial^{2} T_{0}}{\partial \eta^{2}}-\left(\frac{3 \lambda N P r}{3 N+4}\right) \frac{\partial T_{0}}{\partial \eta}=0 \\
& \frac{\partial^{2} T_{1}}{\partial \eta^{2}}-\left(\frac{3 \lambda N P r}{3 N+4}\right) \frac{\partial T_{1}}{\partial \eta}-\left(\frac{3 n N P r}{3 N+4}\right) T_{1}=0
\end{aligned}
$$

Subject to the boundary conditions:

$$
\begin{array}{rlrl}
\text { at } & \theta_{0}=1, & \theta_{1}=1, \\
\text { at } & \eta=\alpha ; & \theta_{0}=T_{0}, & \theta_{1}=T_{1}, \\
& \frac{\partial \theta_{0}}{\partial \eta}=\frac{\partial T_{0}}{\partial \eta}, & \frac{\partial \theta_{1}}{\partial \eta}=\frac{\partial T_{1}}{\partial \eta},
\end{array}
$$

at $\quad \eta=1 ; \quad T_{0}=0, \quad T_{1}=0$

Solving (17) to (21), we obtain

$$
\begin{aligned}
& \theta_{0}=\frac{e^{a_{1} \eta}-e^{a_{1}}}{1-e^{a_{1}}} \\
& \theta_{1}=\frac{e^{a_{3}+a_{4} \eta}-e^{a_{1} \eta+a_{4}}}{e^{a_{3}}-e^{a_{4}}} \\
& T_{0}=\frac{e^{a_{1} \eta}-e^{a_{1}}}{1-e^{a_{1}}} \\
& T_{1}=\frac{e^{a_{3}+a_{4} \eta}-e^{a_{1} \eta+a_{4}}}{e^{a_{3}}-e^{a_{4}}}
\end{aligned}
$$

\subsection{Velocity Distribution}

Let $\bar{F}=U+i V$, and $F=u+i v$

Using above and relevant boundary conditions, equations (8), (9) and (11), (12) reduce to

$$
\begin{aligned}
\frac{\partial \bar{F}}{\partial t}+\lambda \frac{\partial \bar{F}}{\partial \eta}+2 i R e \bar{F} & \\
& =\frac{\partial^{2} \bar{F}}{\partial \eta^{2}}-k\left(\frac{\partial^{3} \bar{F}}{\partial t \partial \eta^{2}}+\lambda \frac{\partial^{3} \bar{F}}{\partial \eta^{3}}\right) \\
& +G r(\theta-1)-\left(\frac{1}{K}+M^{2}\right) \bar{F}
\end{aligned}
$$

$\frac{\partial F}{\partial t}+\lambda \frac{\partial F}{\partial \eta}+2 i R e F$

$$
\begin{aligned}
& =\frac{\partial^{2} F}{\partial \eta^{2}}-k\left(\frac{\partial^{3} F}{\partial t \partial \eta^{2}}+\lambda \frac{\partial^{3} F}{\partial \eta^{3}}\right) \\
& +G r(T-1)-M^{2} F
\end{aligned}
$$

the transformed boundary conditions are:

$$
\begin{array}{ll}
\text { at } \quad \eta=0 ; & \bar{F}=0 \\
\text { at } \eta=\alpha ; & \bar{F}=F, \\
& \frac{\partial \bar{F}}{\partial \eta}=\frac{\partial F}{\partial \eta} \\
\text { at } \eta=1 ; & F=0,
\end{array}
$$

No analytical solution can be expected from the equation (27) and (28), so a regular perturbation scheme has been developed by expanding $\mathrm{U}, \mathrm{V}, \mathrm{u}$ and $\mathrm{v}$ as:

$$
\begin{aligned}
& U(\eta, t)=U_{0}(\eta)+\varepsilon U_{1}(\eta) e^{n t} \\
& V(\eta, t)=V_{0}(\eta)+\varepsilon V_{1}(\eta) e^{n t} \\
& u(\eta, t)=u_{0}(\eta)+\varepsilon u_{1}(\eta) e^{n t} \\
& v(\eta, t)=v_{0}(\eta)+\varepsilon v_{1}(\eta) e^{n t}
\end{aligned}
$$


so that

$$
\begin{aligned}
& \bar{F}(\eta, t)=U_{0}(\eta)+i V_{0}(\eta)+\varepsilon\left[U_{1}(\eta)+i V_{1}(\eta)\right] e^{n t} \\
& =\bar{F}_{0}(\eta)+\varepsilon \bar{F}_{1}(\eta) e^{n t} \\
& \\
& F(\eta, t)=u_{0}(\eta)+i v_{0}(\eta)+\varepsilon\left[u_{1}(\eta)+i v_{1}(\eta)\right] e^{n t} \\
& =F_{0}(\eta)+\varepsilon F_{1}(\eta) e^{n t}
\end{aligned}
$$

Substituting (31) in (27)-(29) and comparing the coefficients of $e^{n t}$, we obtain

$$
\begin{aligned}
& \frac{\partial^{2} \bar{F}_{0}}{\partial \eta^{2}}-\lambda \frac{\partial \bar{F}_{0}}{\partial \eta}-k \lambda \frac{\partial^{3} \bar{F}_{0}}{\partial \eta^{3}}-b_{1} \bar{F}_{0}+G r\left(\theta_{0}-1\right)=0 \\
& \frac{\partial^{2} F_{0}}{\partial \eta^{2}}-\lambda \frac{\partial F_{0}}{\partial \eta}-k \lambda \frac{\partial^{3} F_{0}}{\partial \eta^{3}}-b_{3} F_{0}+G r\left(T_{0}-1\right)=0 \\
& \frac{\partial^{2} \bar{F}_{1}}{\partial \eta^{2}}-\lambda \frac{\partial \bar{F}_{1}}{\partial \eta}-k\left(n \frac{\partial^{2} \bar{F}_{1}}{\partial \eta^{2}}+\lambda \frac{\partial^{3} \bar{F}_{1}}{\partial \eta^{3}}\right)-b_{2} \bar{F}_{1}+\operatorname{Gr} \theta_{1} \\
& =0 \\
& \frac{\partial^{2} F_{1}}{\partial \eta^{2}}-\lambda \frac{\partial F_{1}}{\partial \eta}-k\left(n \frac{\partial^{2} F_{1}}{\partial \eta^{2}}+\lambda \frac{\partial^{3} F_{1}}{\partial \eta^{3}}\right)-b_{4} F_{1}+G r T_{1} \\
& =0
\end{aligned}
$$

and the corresponding boundary conditions are

$$
\begin{array}{lll}
\text { at } \eta=0 ; & \bar{F}_{0}=0 & \bar{F}_{1}=0 \\
\text { at } \eta=\alpha ; & \bar{F}_{0}=F_{0}, & \bar{F}_{1}=F_{1} \\
& \frac{\partial \bar{F}_{0}}{\partial \eta}=\frac{\partial F_{0}}{\partial \eta}, & \frac{\partial \bar{F}_{1}}{\partial \eta}=\frac{\partial F_{1}}{\partial \eta}
\end{array}
$$

$$
\text { at } \eta=1 ; \quad F_{0}=0, \quad F_{1}=0
$$

Using multi-parameter perturbation technique and taking $k<<$ 1 (as for small shear rate $k_{1}$ is very small) [Nowinski and Ismail(1965)], we assume

$$
\begin{array}{ll}
\bar{F}_{0}=\bar{F}_{00}+k \bar{F}_{01}, & F_{0}=F_{00}+k F_{01}, \\
\bar{F}_{1}=\bar{F}_{10}+k \bar{F}_{11} & F_{1}=F_{10}+k F_{11}
\end{array}
$$

Substituting (37) in the equations (32)-(35) and equating the coefficients of the same degree terms and neglecting terms of $O\left(k^{2}\right)$, the following differential equations are obtained:

$$
\begin{aligned}
& \frac{\partial^{2} \bar{F}_{00}}{\partial \eta^{2}}-\lambda \frac{\partial \bar{F}_{00}}{\partial \eta}-b_{1} \bar{F}_{00}+\operatorname{Gr}\left(\theta_{0}-1\right)=0 \\
& \frac{\partial^{2} F_{00}}{\partial \eta^{2}}-\lambda \frac{\partial F_{00}}{\partial \eta}-b_{3} F_{00}+\operatorname{Gr}\left(T_{0}-1\right)=0 \\
& \frac{\partial^{2} \bar{F}_{10}}{\partial \eta^{2}}-\lambda \frac{\partial \bar{F}_{10}}{\partial \eta}-b_{2} \bar{F}_{10}+\operatorname{Gr} \theta_{1}=0 \\
& \frac{\partial^{2} F_{10}}{\partial \eta^{2}}-\lambda \frac{\partial F_{10}}{\partial \eta}-b_{4} F_{10}+G r T_{1}=0=0 \\
& \frac{\partial^{2} \bar{F}_{01}}{\partial \eta^{2}}-\lambda \frac{\partial \bar{F}_{01}}{\partial \eta}-b_{1} \bar{F}_{01}=\lambda \frac{\partial^{3} \bar{F}_{00}}{\partial \eta^{3}}
\end{aligned}
$$

$$
\begin{aligned}
& \frac{\partial^{2} F_{01}}{\partial \eta^{2}}-\lambda \frac{\partial F_{01}}{\partial \eta}-b_{3} F_{01}=\lambda \frac{\partial^{3} F_{00}}{\partial \eta^{3}} \\
& \frac{\partial^{2} \bar{F}_{11}}{\partial \eta^{2}}-\lambda \frac{\partial \bar{F}_{11}}{\partial \eta}-b_{2} \bar{F}_{11}=n \frac{\partial^{2} \bar{F}_{10}}{\partial \eta^{2}}+\lambda \frac{\partial^{3} \bar{F}_{10}}{\partial \eta^{3}} \\
& \frac{\partial^{2} F_{11}}{\partial \eta^{2}}-\lambda \frac{\partial F_{11}}{\partial \eta}-b_{4} F_{11}=n \frac{\partial^{2} F_{10}}{\partial \eta^{2}}+\lambda \frac{\partial^{3} F_{10}}{\partial \eta^{3}}
\end{aligned}
$$

subject to boundary conditions

$$
\begin{aligned}
& \text { at } \eta=0 ; \quad \bar{F}_{00}=0, \quad \bar{F}_{01}=0, \quad \bar{F}_{10}=0, \quad \bar{F}_{11}=0, \\
& \text { at } \eta=\alpha ; \quad \bar{F}_{00}=F_{00}, \quad \bar{F}_{01}=F_{01}, \quad \bar{F}_{10}=F_{10}, \quad \bar{F}_{11}=F_{11}, \\
& \frac{\partial \bar{F}_{00}}{\partial \eta}=\frac{\partial F_{00}}{\partial \eta}, \quad \frac{\partial \bar{F}_{01}}{\partial \eta}=\frac{\partial F_{01}}{\partial \eta}, \quad \frac{\partial \bar{F}_{10}}{\partial \eta}=\frac{\partial F_{10}}{\partial \eta}, \quad \frac{\partial \bar{F}_{11}}{\partial \eta}=\frac{\partial F_{11}}{\partial \eta} \\
& \text { at } \eta=1 ; \quad F_{00}=0, \quad F_{01}=0 \quad F_{10}=0 \quad F_{11}=0
\end{aligned}
$$

\section{RESULTS AND DISCUSSION}

The non-dimensional skin friction $\sigma_{0}$ at the plate $\eta=0$ is given by

$$
\sigma_{0}=\frac{\partial U}{\partial \eta}-k\left(\frac{\partial^{2} U}{\partial t \partial \eta}+\lambda \frac{\partial^{2} U}{\partial \eta^{2}}\right)
$$

The non-dimensional skin friction $\sigma_{1}$ at the plate $\eta=1$ is given by

$$
\sigma_{1}=\frac{\partial u}{\partial \eta}-k\left(\frac{\partial^{2} u}{\partial t \partial \eta}+\lambda \frac{\partial^{2} u}{\partial \eta^{2}}\right)
$$

The non-dimensional heat flux $N u_{0}$ at the plate $\eta=0$ in terms of Nusselt number is given by

$$
N u_{0}=\left(\frac{\partial \theta}{\partial \eta}\right)_{\eta=0}=\left(\frac{\alpha_{1}}{1-e^{\alpha_{1}}}\right)+\varepsilon e^{n t}\left(\frac{a_{4} e^{\alpha_{3}}-a_{3} e^{\alpha_{4}}}{e^{\alpha_{3}}-e^{\alpha_{4}}}\right)
$$

The non-dimensional heat flux $N u_{1}$ at the plate $\eta=1$ in terms of Nusselt number is given by

$$
N u_{1}=\left(\frac{\partial T}{\partial \eta}\right)_{\eta=1}=\left(\frac{\alpha_{1} e^{\alpha_{1}}}{1-e^{\alpha_{1}}}\right)+\varepsilon e^{n t+a_{3}+a_{4}}\left(\frac{\alpha_{4}-\alpha_{3}}{e^{\alpha_{3}}-e^{\alpha_{4}}}\right)
$$

where constants are obtained but not given here for the sake of brevity.

The main objective of this study is to bring out the effects of visco-elastic parameter on the governing flow as the effects of other parameters have been discussed in detatil by Chauhan and Rastogi (2010). The visco-elastic effect is exhibited through the non-dimensional parameter $k$. The corresponding results for Newtonian fluid are obtained by setting $k=0$. This study analyzes the configuration in vertical parallel plate channel where a porous substrate is perfectly attached to the left vertical plate. Results are presented graphically and discussed. Motivation of this work lies behind its use in practical situation such as metallurgy where the process of solidification is characterized by the presence of a liquid, a mushy zone and a solid zone, cooling of electronic 
components etc. The fluid velocity and the shearing stress at the plate are illustrated graphically for various values of thermal Grashof number Gr, Prandtl number Pr, magnetic parameter $\mathrm{M}$, Stark number $\mathrm{N}$, radiation parameter $\mathrm{Re}$, injection suction parameter $\lambda$, permeability parameter $\mathrm{K}$ involved in the solution. The values of the parameters $G r=6, P r=6, M=1.41, N=1, R e=5, n=0.2, t=$ $1, \lambda=1, \varepsilon=0.1, K=0.1$ are kept fixed throughout the discussion.

Figures 2 to 15 demonstrate the pattern of fluid velocity against the displacement y for the various values of viscoelastic parameter. Figure 2-6 illustrate the effect of various parameters such as fluid velocity in $\mathrm{x}$-direction.

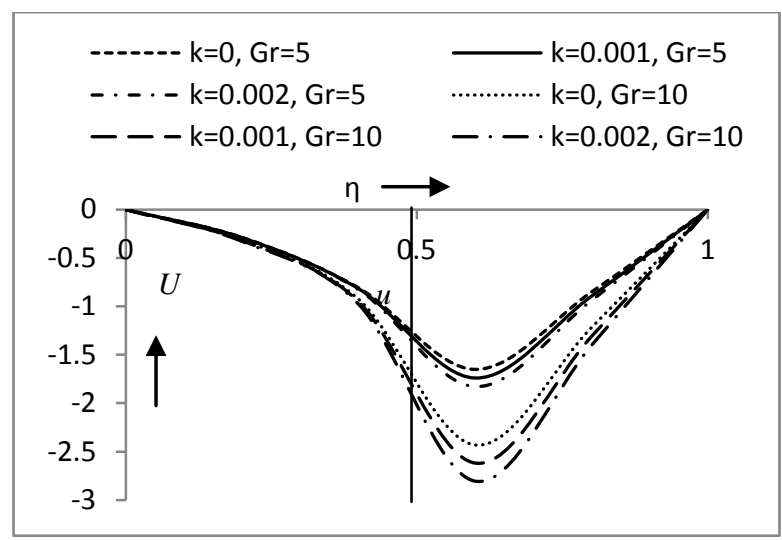

Figure 2. Fluid velocity in $\mathrm{x}$-direction $\mathrm{U}$, u vs $\eta$.

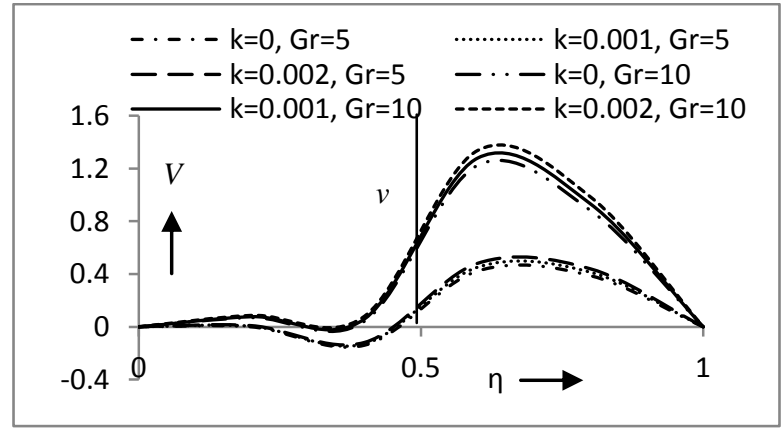

Figure 3. Fluid velocity in y-direction $V, \mathbf{v}$ vs $\eta$.

The effects of Grashof number on fluid velocity in x-direction and y-direction are obtained from figures 2 and 3 respectively. Grashof number signifies the relative effect of the thermal buoyancy force to the viscous hydrodynamic force in the boundary layer. In our study, we have considered the positive values of Grashof number. Gr > 0 , interprets that flow past an externally cooled plate. The figure 2 states that the rising value of Grashof number enhances the viscosity of both Newtonian and non-Newtonian fluids, which in turn drop off the speeds of both the fluids in $\mathrm{x}$ - direction. But in $\mathrm{y}$-direction speed of the fluid first diminishes slightly and then boost up to a considerable amount.

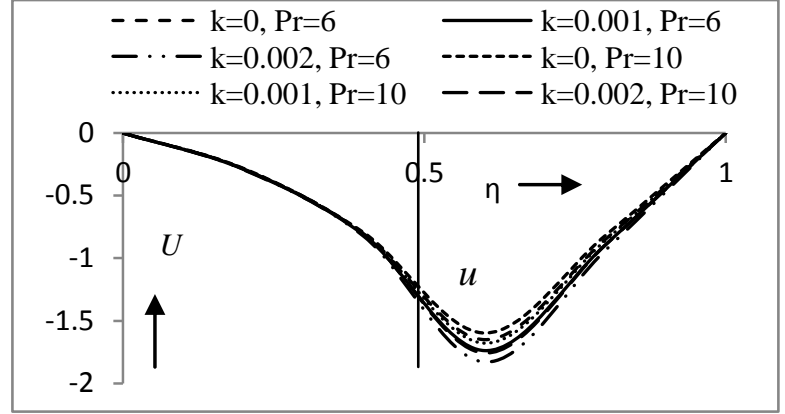

Figure 4. Fluid velocity in $x$-direction $U$, $u$ vs $\eta$.

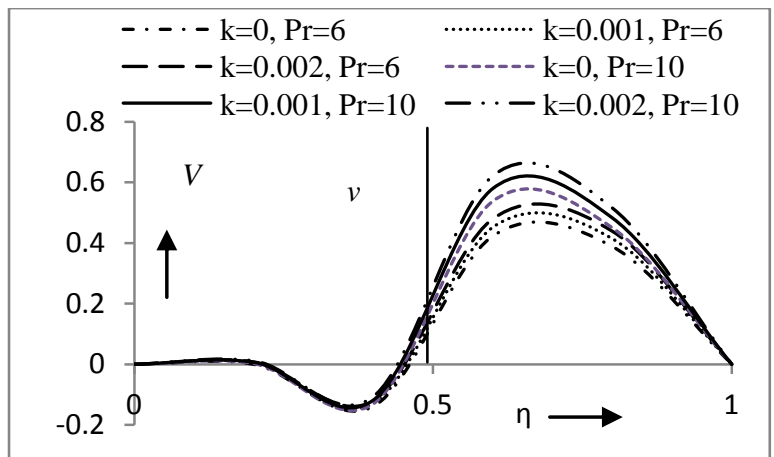

Figure 5. Fluid velocity in y-direction $V, v$ vs $\eta$.

Influence of Prandtl number on the primary and secondary flows has been illustrated in Figures 4 and 5, keeping all other parameters fixed. It explains the relative effectiveness of momentum diffusion and thermal diffusion in the fluid flow. It is observed that the rising value of Prandtl number diminishes the primary flow in the porous substrate attached to the left hot wall and its nearby region whereas magnitude accelerates near the other wall. Again for secondary flow fluid velocity enhances and then comes down.

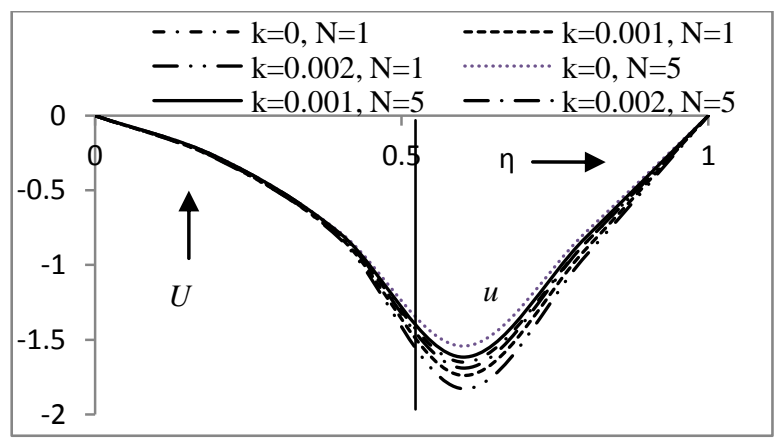

Figure 6. Fluid velocity in $x$-direction $U$, $\mathbf{u}$ vs $\eta$.

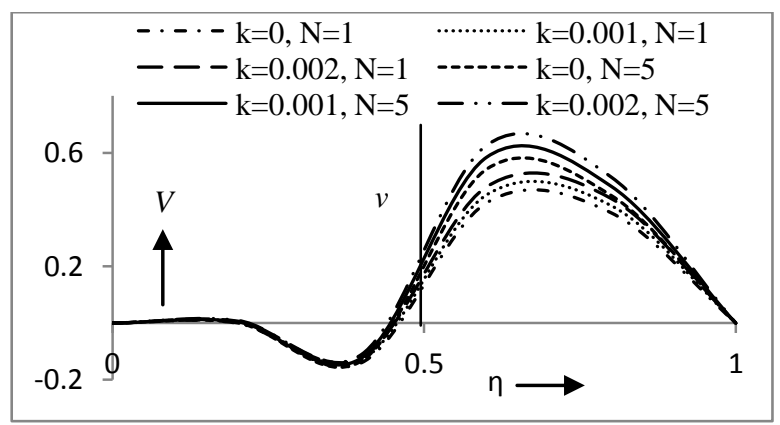

Figure 7. Fluid velocity in y-direction $V, \mathrm{v} v \mathrm{vs} \eta$. 
The influence of thermal radiation can be explained through the Stark number N, which is defined as relative contribution of the conduction heat transfer to the thermal radiation transfer. It is seen in Figures 6 and 7 that by increasing values of $\mathrm{N}$ the primary and secondary flow decreases near the left wall in the porous medium. Also flow pattern remains same for both Newtonian and non-Newtonian fluid.

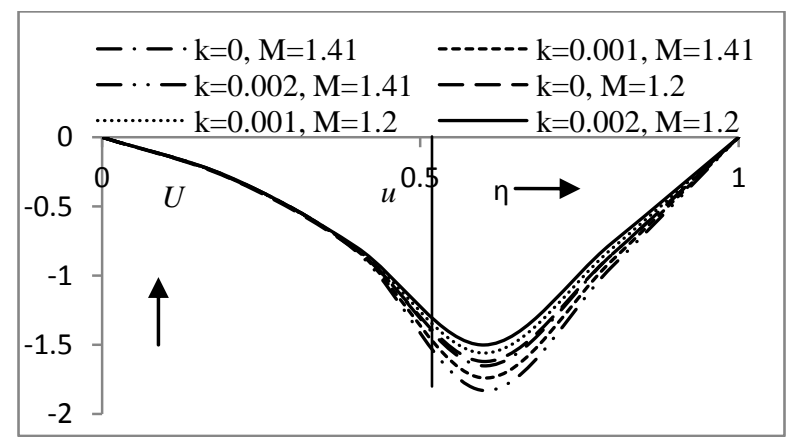

Figure 8. Fluid velocity in $\mathrm{x}$-direction $\mathrm{U}$, u vs $\eta$.

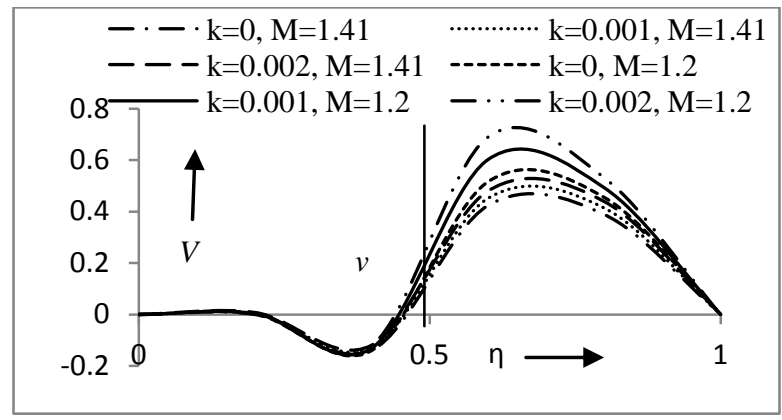

Figure 9. Fluid velocity in y-direction $\mathrm{V}, \mathrm{v}$ vs $\eta$.

Figures 8 and 9 illustrate the behaviour of fluid flow for various values of Hartmann number. Hartmann number depicts the strength of transverse magnetic field. The application of transverse magnetic field produces Lorentz force and the Lorentz force has a retarding effect on the velocity. As a consequence, the thickness of the fluid will be enlarged and the speed will go down. This diminishing trend in speed is observed in visco-elastic fluid characterized by Walters liquid (Model B' ).

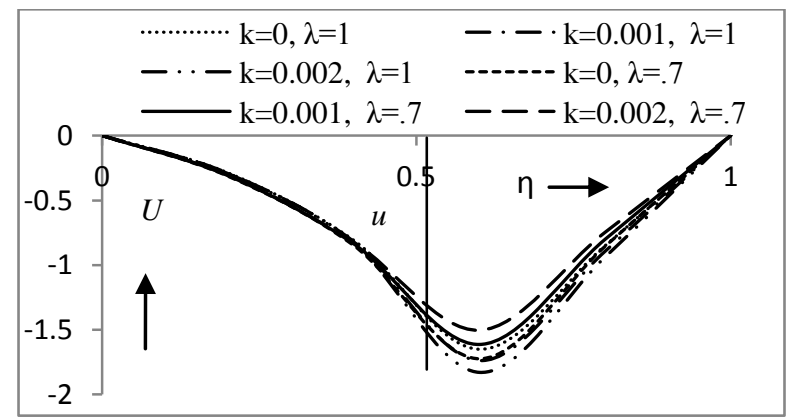

Figure 10. Fluid velocity in $x$-direction $U$, u vs $\eta$.

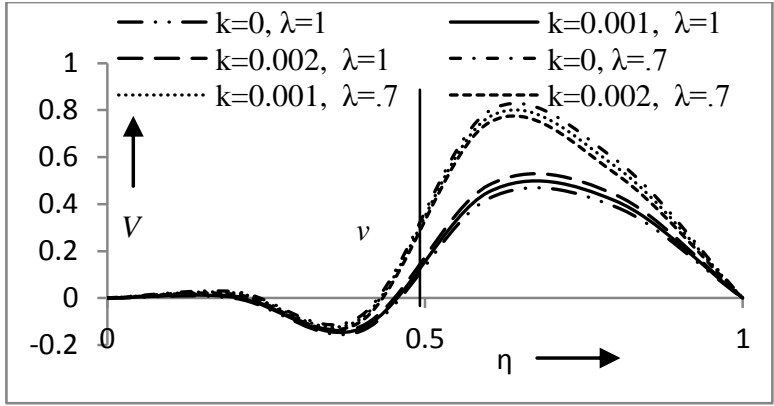

Figure 11. Fluid velocity in y-direction $V, \mathbf{v}$ vs $\eta$.

Figures 10 and 11 represent the primary and secondary flow patterns with the increasing values of suction injection parameter $\lambda$. Temperature of the heated wall (left) at $\mathrm{z}=0$ is a function of time, as given by in the boundary conditions and the cooled wall at $\mathrm{z}=\mathrm{d}$ is maintained at a constant temperature. Further it was assumed that the temperature difference is sufficiently small so that the density changes of the fluid in the system will be small. When the injection suction parameter $\lambda$ is positive, fluid is injected through the hot wall into the channel and sucked out through the cold wall. It is observed that in case of secondary flow velocity enhances with the increasing values of $\lambda$.

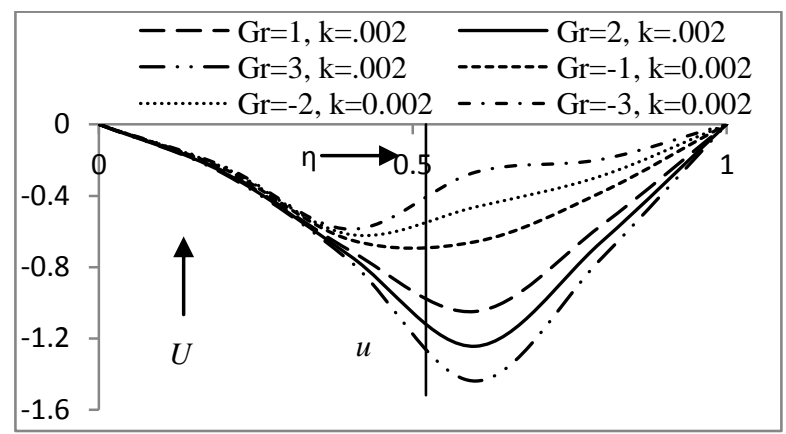

Figure 12. Fluid velocity in $\mathrm{x}$-direction $\mathrm{U}$, u vs $\eta$.

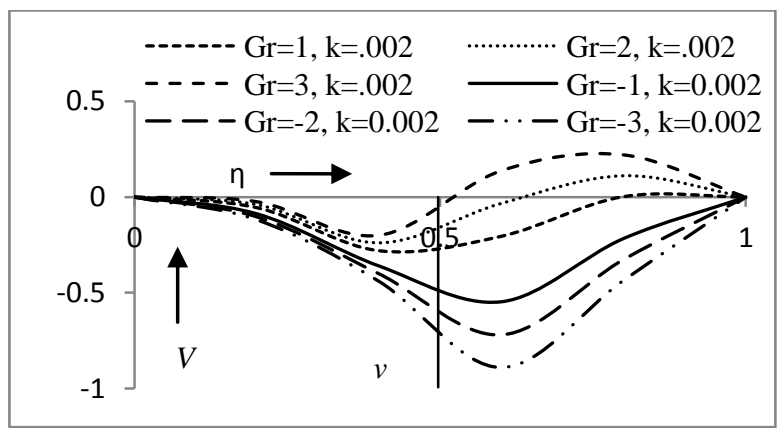

Figure 13. Fluid velocity in y-direction $V, \mathbf{v}$ vs $\eta$.

For different values of the Grashof number Gr the pattern of fluid velocity for visco-elastic fluid is plotted in figures 12 and 13. It is noticed that for hot plate $(\mathrm{Gr}<0)$ both primary and secondary velocity follows a downward trend and then move up. For cooled plate $(\mathrm{Gr}>0)$ fluid velocity increases in comparison with hot plate. 


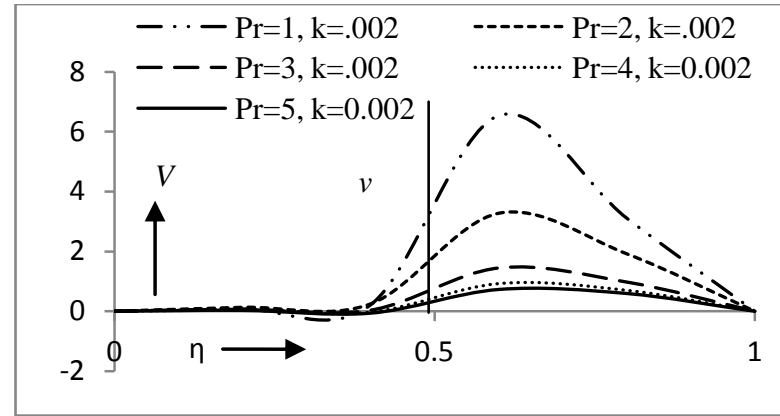

Figure 14. Fluid velocity in y-direction $V$, v vs $\eta$.

Figure 14 depicts the secondary fluid velocity pattern for various values of Prandtl number. We can see that with the development of Prandtl number fluid velocity diminishes. The rising value of Prandtl number raises the thickness of the fluid and hence the fluid experiences a decelerating trend. This physical phenomenon is observed in visco-elastic fluid.

The study of skin friction for the concerned flow is very important from practical point of view. Figures 15-20 depict the the effect of viscous drag on Newtonian and visco-elastic fluid flow phenomenon.

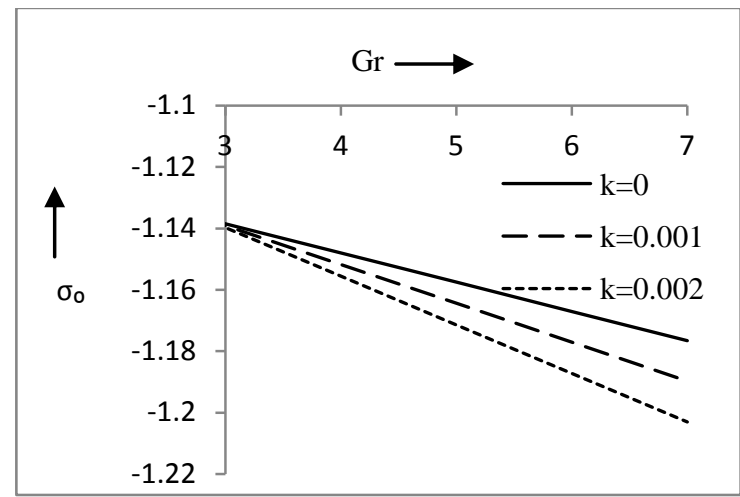

Figure 15. Skin friction $\sigma_{0}$ vs Gr.

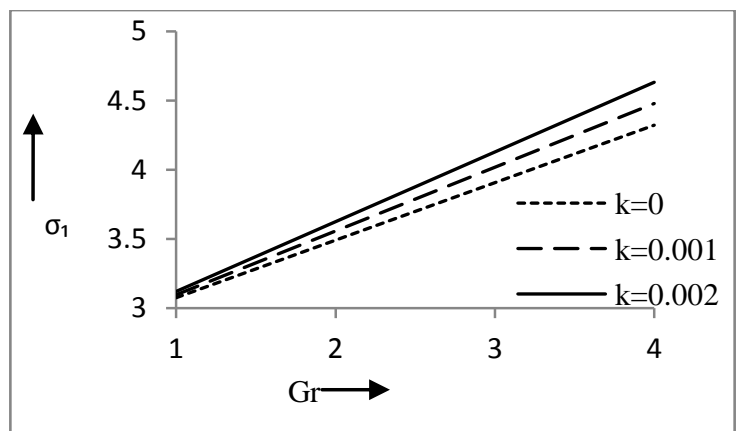

Figure 16. Skin friction $\sigma_{1}$ vs Gr.

Figures 15 and 16 illustrate the variations of the skin friction at the hot wall $\sigma_{0}$ and at the cold wall $\sigma_{1}$.It is found that at the hot wall skin friction decreases in magnitude but at the cold wall it enhances by increasing the thermal Grashof number Gr.

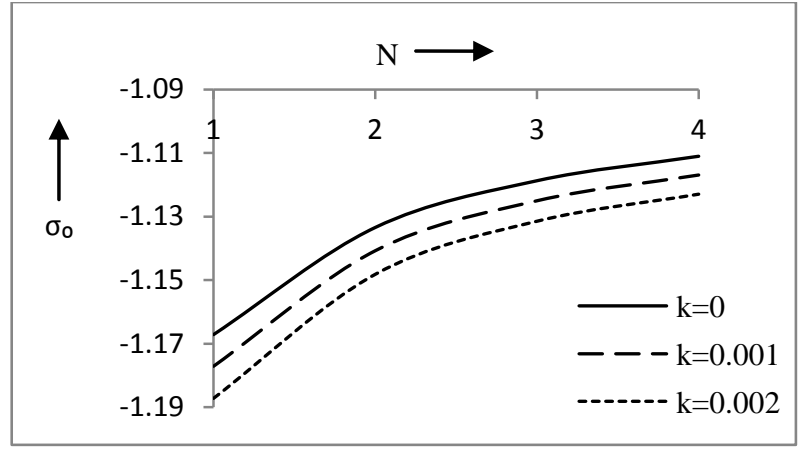

Figure 17. Skin friction $\sigma_{0}$ vs $\mathrm{N}$.

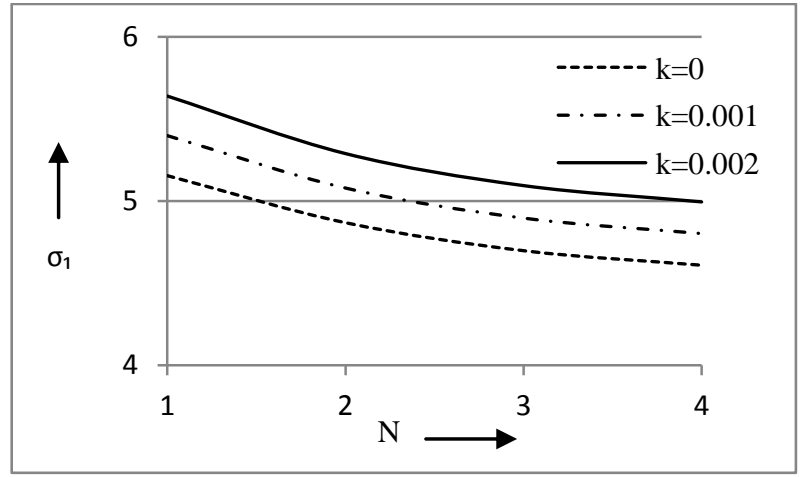

Figure 18. Skin friction $\sigma_{1}$ vs $\mathrm{N}$.

Figures 17 and 18 exemplify the skin friction for various values of suction injection parameter $\mathrm{N}$. It is observed that viscous drag enhances near the hot plate and diminishes near the cold plate. Both simple as well as non-Newtonian fluid follow the matching movement.

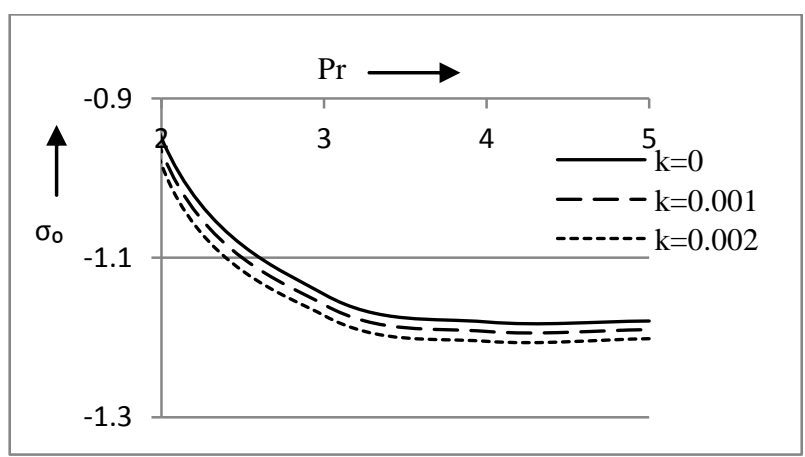

Figure 19. Skin friction $\sigma_{0}$ vs $\mathrm{Pr}$.

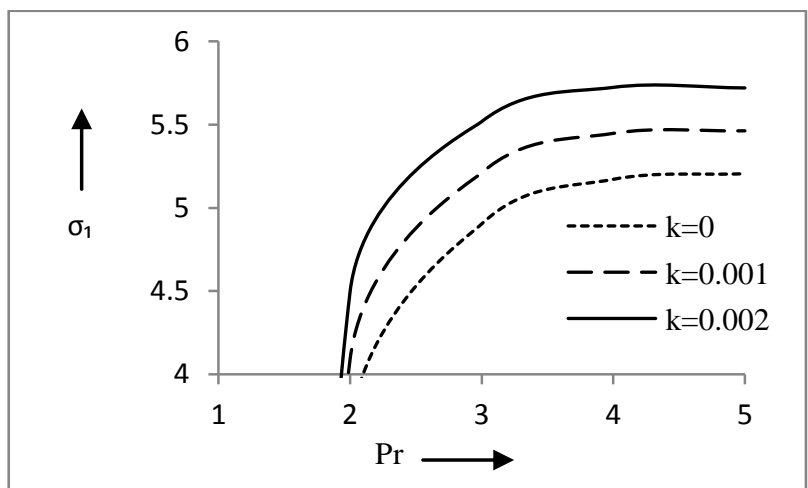

Figure 20. Skin friction $\sigma_{1}$ vs $\operatorname{Pr}$ 
Figures 19 and 20 show the behaviour of skin friction against Prandtl number for Newtonian as well as non-Newtonian fluid flows. It is noticed that in the neighbourhood of small values of Prandtl number, the shearing stress subdues near the hot plate $\sigma_{0}$ but it shows a rising trend near the cold plate $\sigma_{1}$. This phenomenon is observed in visco-elastic fluid as well as in simple Newtonian fluid.

\section{CONCLUSIONS}

The problem of unsteady, MHD elastico-viscous flow past a vertical parallel plate where a porous substrate is attached to the left vertical palate has been studied. The velocity and temperature fields are obtained analytically. Graphical results are presented and discussed for various physical parametric values. The main findings are as follows:

- The primary fluid flow is decelerated and then accelerated slowly during the enhancement of viscoelastic parameter.

- The secondary fluid flow is accelerated and then retarded slowly during the enhancement of viscoelastic parameter.

- The fluid slowed down with the increasing values of visco elastic parameter in comparison with the Newtonian fluid in case of primary velocity but a reverse trend is observed for secondary fluid flow velocity.

- The viscous drag formed by both Newtonian and nonNewtonian fluid will experience a decreasing trend during the growth of solutal Grashof number $\mathrm{Gr}$ and Prandtl number Pr near the cooled plate $\sigma_{1}$.

- An accelerating trend in viscous drag is observed in case of flow past an heated plate for both Newtonian and non-Newtonian fluid for increasing values of Stark number N

- An accelerating trend in shearing stress is observed in case of flow past an cooled plate for both Newtonian and non-Newtonian fluid.

- The rate of heat transfer will not differ significantly during the various values of visco-elastic parameter.

\section{REFERENCES}

[1] Aung, W., Fletcher, L. S., and Sernas, V. 1972. Developing laminar free convection between vertical flat plates with asymmetric heating. Int. J. Heat Mass Transfer. 15, 2293-2304.

[2] Burch, T., Rhodes T. and Acharya, S. 1985. Laminar natural convection between finitely conducting vertical plates. Int. J. Heat Mass Transfer. 28, 1173-1186.

[3] Buhler, K. 2003. Special solutions of the Boussinesq equations for free convection flows in a vertical gap, Heat Mass Transfer. 39, 631-638.

[4] Weidman, P. D. and Medina A. 2008. Porous media convection between vertical walls: Continuum of solutions from capped to open ends. Acta Mech. 199, 209-216.

[5] Sanyal, D. C. and Adhikari, A. 2006. Effects of radiation on MHD vertical channel flow. Bull. Cal. Mathe. Soc. $98,5,487-497$
[6] Chauhan, D. S. and Jain, R. 2005. Three dimensional MHD steady flow of a viscous imcompressible fluid over a highly porous layer. Modelling, Measurement and Control B. 74, 5, 19-34.

[7] Magyari, E. 2007. Normal mode analysis of the fully developed free convection flow in a vertical slot with open to capped ends. Heat Mass Transfer. 43, 827-832.

[8] Yih, K. A. 1998. The effect of uniform suction/blowing on heat transfer of magnetohydrodynmic Hiemenz flow through porous media. Acta Mechanica. 130, 147-158.

[9] Al-Nimr, M. A. and Haddad, O. H. 1999. Fully developed free convection in open-ended vertical channels partially filled with porous material. J. Porous Media. 2, 179-189.

[10] Alkam, M. K., Al-Nimr, M. A., Hamdan, M. O. 2002. On forced convection in channels partially filled with porous substrates. Heat and Mass Transfer. 38, 337-342.

[11] Weidman, P. D. 2006. Convection regime flow in a vertical slot: Continuum of solutions from capped to open ends. Heat Mass Transfer. 43, 103-109.

[12] Kim, S. H., Anand N. K. and Aung, W. 1990. Effect of wall conduction on free convection between asymmetrically heated vertical plates. Uniform wall heat flux. Int. J. Heat Mass Transfer. 33, 1013-1023.

[13] Bian, W., Vasseur, P. Bilgen, E. and Meng, F. 1996. Effect of an electromagnetic field on natural convection in an inclined porous layer. Int. J. Heat Fluid Flow. 17, $36-44$.

[14] Chang, W. J. and Chang, W. L. 1996. Mixed convection in a vertical parallel plate channel partially filled with porous media of high permeability. Int. J. Heat Mass Transfer. 39, 1331-1342.

[15] Al-Nimr, M. A. and Khadrawi, A. F. 2003. Transient free convection fluid flow in domains partially filled with porous media. Transport in porous media. 51, 157-172.

[16] Chauhan, D. S. and Rastogi, P. 2010. Radiation effects on natural convection MHD flow in rotating vertical porous channel partially filled with a porous medium. Appl. Math. Sci. 4, 13, 643-655.

[17] Subhas Abel, M. Joshi, A. and Sonth, R.M. 2001. Heat transfer in MHD visco-elastic fluid flow over a stretching surface. Zeitschrift für angewandte Mathematikund Mechanik. 81, 19, 691-698.

[18] Sonth, R. M., Khan, S.K., Subhash, A. M and Prasad, K. V. 2002. Heat and mass transfer in a visco-elastic fluid flow over an accelerating surface with heat source/sink and viscous dissipation. Heat Mass Transfer. $38,3,213-220$

[19] Abel, M. S., Siddheswar, P.G., Nandeeppanavar, M. M. 2007. Heat transfer in a viscoelastic boundary layer flow over a stretching sheet with viscous dissipation and nonuniform heat source. Int. J. Heat Mass Transfer. 50, 5-6, 960-966.

[20] Choudhury, R. and Das, A. 2002. Steady flow of Walters liquid $\mathrm{B}^{\prime}$ through the annulus of porous coaxial circular cylinders. Ind J. Pure Appl. Maths. (IJPAM). 33, 6, 807817. 
[21] Choudhury, R. and Dey, D. 2010. Free convective viscoelastic flow with heat and mass transfer through a porous medium with periodic permeability. Int. J. of Heat Mass Transfer. (Elsevier). 53, 1666-1672.

[22] Choudhury, R. and Deb, H. R. 2011. Flow of an elasticoviscous fluid due to a rotating disk in presence of an infinite saturated porous medium. Int. J. fluid engg. 3, 2, 187-196.

[23] Choudhury, R. and Das, U. J. 2012. Heat transfer to MHD oscillatory visco-elastic flow in a channel filled with porous medium. Phy. Res. Int. Egypt. Ar ID 879537,5 pages.

[24] Choudhury, R. and Das, U. J. 2013. Visco-elastic effects on the three dimensional hydrodynamic flow past a vertical porous plate. Int. J. Heat Tech. Italy. 31,1, 1-8.

[25] Walters, K. 1960. The motion of an elastico-viscous liquid contained between co-axial cylinders (II). Quart. J. Mech. Appl. Math. 13, 444-461.
[26] Walters, K. 1962. The solution of flow problems in the case of materials with memories. J. Mecanique. 1, 473478

[27] Cowling, T. G. 1957. Magnetohydrodynamics. Interscience Publishers. N. Y.

[28] Siegel, R. and Howell, J. R. 1972. Thermal Radiation Heat Transfer, International Student Edition. Mc GrawHill. New York.

[29] Raptis, A. 1998. Radiation and free convection flow through a porous medium. Int. Commun. Heat Mass Transfer. 25, 289-295.

[30] Nowinski, J. L. and Ismail, I. A. 1965. Application of a multi-parameter perturbation method to elastostatics in development in theoretical and applied mechanics. N.A. Shaw. vol. 11. Pergamon Press. Oxford. 35. 